\title{
Simulating the Dominant Effect of a Few Critical Sites on Supply Chains Using the Inter-industry Relations Table
}

\author{
Sadao Suganuma and Masaru Nakano \\ The Graduate School of System Design and Management, Keio University \\ Kyosei Building, 4-1-1 Hiyoshi, Kohoku-ku, Yokohama \\ Kanagawa 223-8526, Japan \\ suganuma235@a6.keio.jp, nakano@sdm.keio.ac.jp
}

\begin{abstract}
We study supply chain disruptions, particularly in the passenger vehicles sector, caused by the mass industrial shutdowns due to the Great East Japan Earthquake in March, 2011, along with the restoration process to assess supply chain resiliency. We first investigate the extent of damage using the inter-industry relations table and then analyze from the viewpoints of geography, time, and economy. Following this, the layered structure was derived and the rate-determining factors of the restoration process were identified. Using these factors, we simulate the disruptions in and restoration of the passenger vehicles sector. This method using the I-O table is effective for assessment of supply chain resiliency.
\end{abstract}

Keywords: Supply chain disruptions, Sustainable manufacturing, Supply chain, Risk management, Inter-industry relations, I-O table, Automotive industry, Great East Japan Earthquake.

\section{Introduction}

Supply chains have recently emerged as complex global networks. As such, large natural disasters-such as the 2010 Icelandic volcano eruption, the 2011 Thailand floods, and the Great East Japan Earthquake of 2011-often cause global supply chain disruptions. Accordingly, supply chains need to be assessed to ensure resiliency and the ability to recover quickly. However, few such studies exist (1-2). The goal of this study is to establish a way of assessing supply chain resiliency.

In this paper, we study the supply chain disruptions caused by the Great East Japan Earthquake of 2011, particularly focusing on the passenger vehicles sector, which we take as a representative example of complex global supply chains. The automotive industry suffered considerably due to that disaster. Plants located in the affected regions comprised $30 \%$ of the supply chain; these were taken offline suddenly, disrupting automotive production in the world for several months. Efforts were made to resume production in other areas but initiating operations took much time. Damage assessment was made using various reports, newspapers, etc. (3-8). The main factors 
were analyzed from the viewpoints of geography, time, and economy. We focus on the passenger vehicles sector owing to the complex nature and large size of its supply chain.

The input-output (I-O) table is a statistical table developed by American economist Wassily Leontief. This table has been used in various business fields, for example, in measuring the effectiveness of a policy, ascertaining the industrial structure, analyzing employment patterns, studying the globalization of industrial linkages, analyzing the environmental effect, and so on (10-11). There exist works investigating the economic influence of natural disasters (12-14) using the I-O table: these works study the impact of disasters on a region and industries from multiple viewpoints. We have noted that the regional inter-industry relations table provided by the Japanese government after surveying industries across the country gives quantitative details of the trade between industrial sectors across several districts. Using the inter-industry relations table (henceforth, the I-O table; 9), the layered structure of the vast and complex automotive supply chain was derived. The process was simulated to assess the disruption in and restoration of the supply chain due to a specific sector in a specific area that is deemed a critical sector, going offline.

\section{Methodology}

We focus on the recovery process of the supply chain in the passenger vehicles sector following the disruption caused by the Great East Japan Earthquake, and perform a simulation using the I-O table. Damage assessment was made using various reports by automotive manufacturers and by the Japan Automobile Manufacturers Association, general newspapers and industrial newspapers, etc.

\subsection{Damage to and Recovery of the Passenger Vehicles Sector in 2011}

Fig. 1 shows the monthly car production in Japan. In the figure, the drastic production declines following the Lehman Brothers' bankruptcy and the Great East Japan Earthquake can be easily seen. Comparing the two, the former had a long recovery time as it was a "demand shock," while the latter saw faster recovery as it was a "supply disruption."

\subsection{Interregional I-O Table}

Using the interregional I-O table published by the Ministry of Economy, Trade, and Industry every five years, we analyze the effect of the supply disruptions in East Japan on the firms in other areas.

Table 1 details the interregional I-O table in terms of areas and sectors. We use the latest edition (year 2005) and show the data for nine areas and 53 industrial sectors. The areas were integrated into disaster (Tohoku and Kanto) and non-disaster areas (Others). Fig. 2 (a) gives the earthquake-intensity and devastation maps for the Great East Japan Earthquake, and Fig. 2 (b) provides a map detailing the interregional I-O table. 


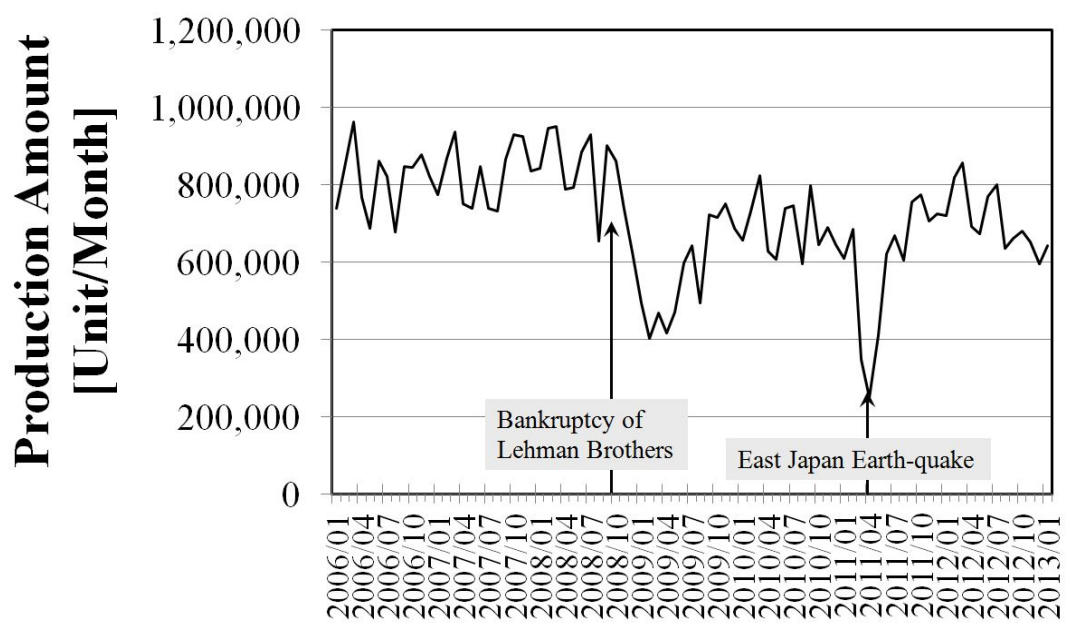

Date

Fig. 1. Impact of the Great East Japan Earthquake on Monthly Passenger Vehicles Production

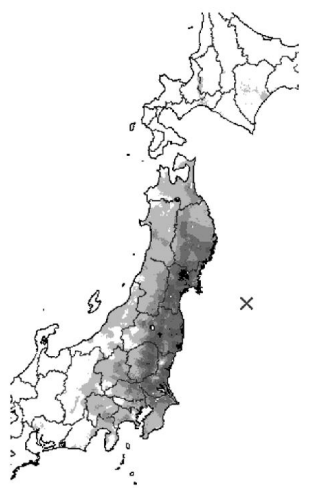

(a)

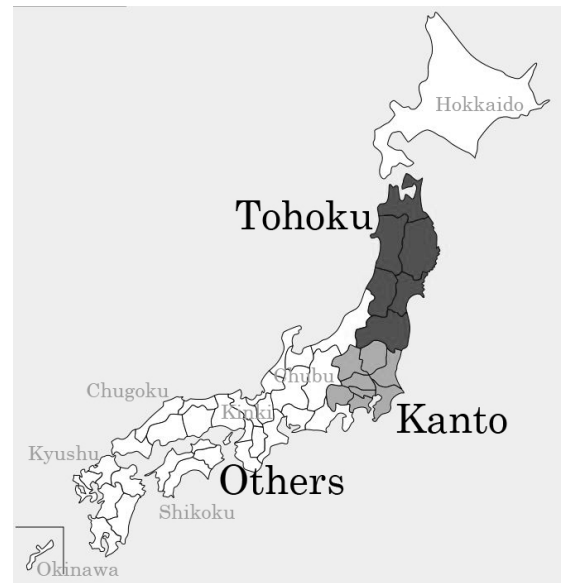

(b)

Fig. 2. Devastation map: (a) Earthquake-intensity map; (b) Map detailing the Interregional I-O Table 
Table 1. Structure of intermediate sectors in the interregional I-O table

\begin{tabular}{|c|c|c|c|c|}
\hline & \multicolumn{3}{|c|}{ Demand Sector } \\
\hline & & Other Areas & Tohoku Area & Kanto Area \\
\hline \multirow{3}{*}{$\begin{array}{l}\text { Supply } \\
\text { Sector }\end{array}$} & Other Areas & $53 \times 53$ sectors & $53 \times 53$ sectors & $53 \times 53$ sectors \\
\hline & Tohoku Area & $53 \times 53$ sectors & $53 \times 53$ sectors & $53 \times 53$ sectors \\
\hline & Kanto Area & $53 \times 53$ sectors & $53 \times 53$ sectors & $53 \times 53$ sectors \\
\hline
\end{tabular}

The industrial sectors were rearranged based on level of integration, common materials used, level of importance for normal economic functioning, and energy consumed.

The transaction spread for all 53 sectors in the integrated areas is given in Fig. 3 (a), and that for the passenger vehicles sector is given in Fig. 3 (b).

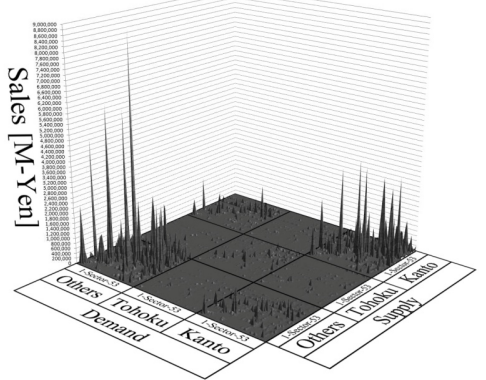

(a)

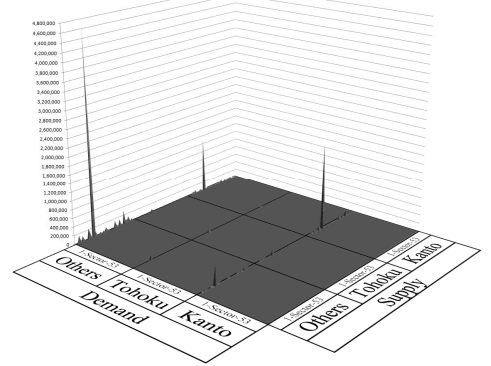

(b)

Fig. 3. Transaction spread for the three areas: (a) All sectors; (b) Passenger vehicles sector

The above figure shows the amount of business between the demand-side sectors and the supply-side sectors. Both sectors have three blocks: Kanto, Tohoku, and others. Trade within Kanto and others is large as the industries here are integrated. The passenger vehicles sector industries are extracted in Fig. 3 (b).

\subsection{Layered Structure of the Passenger vehicles Industry}

In Fig. 4, the 6-layered structure of the supply chain is shown. The passenger vehicles sector is defined as tier 1 (T1), the components and subcomponents industries as tier 2 (T2), and so on. The square matrix in the figure gives the demand for T2 by T1, and is called the L1 trade matrix. We call it as layer 1 (L1). The linear matrix of the sum of demand from each L1 supply-side sector is called the L1 output matrix, and is the L2 demand matrix. The L2 trade matrix is calculated using the L2 demand matrix and the input coefficients matrix.

We thus obtain the 6-layered structure of the supply chain. Fig. 4 shows the trade matrix for each layer of the supply chain at equilibrium, and is also referred to as the 3-D trade amount table. 


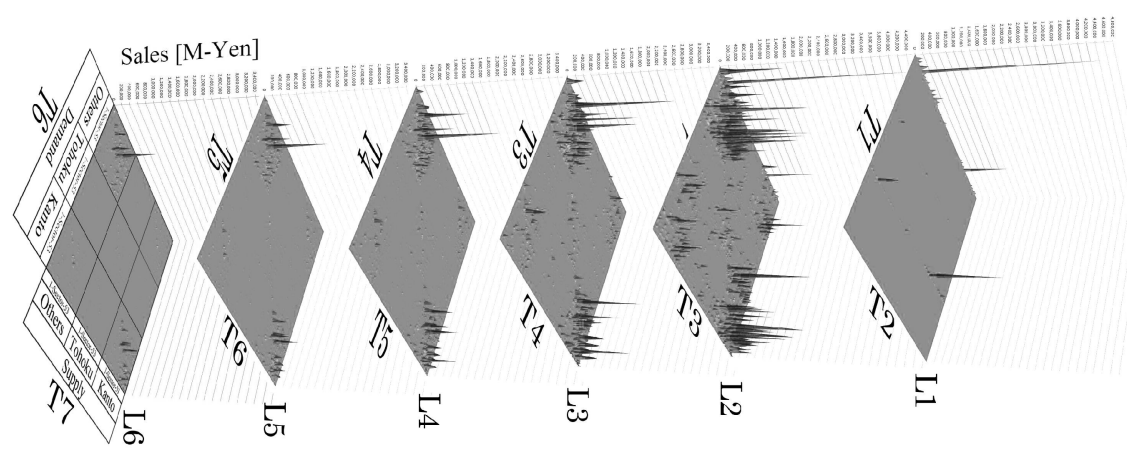

Fig. 4. Layered structure of the supply chain (3-D trade amount table)

In this way, the layered structure of the supply chain is completed and illustrated in Fig. 4. T1 on L1 covers the passenger vehicles manufacturers. T2 on L1 covers the suppliers who provide manufacturers with the parts; these same suppliers form the demand-side sectors that are supplied with parts by T3 industries in L2. As we move from L1 to L6, dominant trades shift to sectors of small parts and raw materials.

\section{Results}

As each supplier prepares its production capacity for the assigned amount, the 3-D trade amount table can be regarded as a 3-D structured capacity table. Although capacity varies with the amount of trade and vice versa, the 3-D structured capacity value is the value when the demand for the final product equals its production. The 3-D structured capacity table is converted to a normalized table named the 3-D utilization table. In the normalized table, in case of a trade at the cross-point of a supply-side sector and a demand-side sector, the value assigned is 1 . Otherwise, no value is assigned. This table thus gives all cross-points at which trades were made.

In this table, the values of all trading sites are 1 under equilibrium, and decrease to 0 if total disruption is observed. The data were collected using various reports, newspapers, etc., and the operation status of each sector in each tier was determined and marked. We thus created a 3-D recurrence equation matrix to express the disruption ripple effect from the lower to upper tiers. The final industrial production level was given by the completion of that matrix.

Each demand sector's procurement rate is given by the lowest availability factor of its supply sectors. The dominant procurement rate of a T1 manufacturer is thus obtained using this sequence, and its total production in that period is then obtained by multiplying procurement rate and gross equilibrium output. Similarly, the production recovery profile is obtained. The simulation results for the disruption in and recovery of the passenger vehicles sector are given in Fig. 5. 


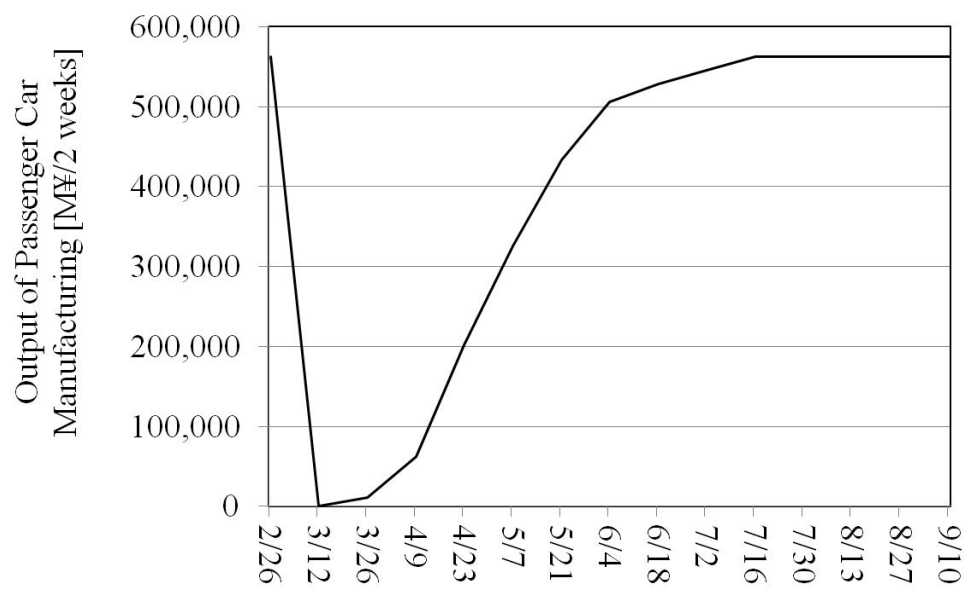

Fig. 5. Disruption in and recovery of the passenger vehicles sector

The Great East Japan Earthquake occurred on March 11, 2011. Fig. 5 shows that the output of passenger vehicles became zero on March 12, reached 100,000 after a month, 300,000 after two months, and 500,000 in June. Actually, the output fell to about 250,000 but did not become zero when the earthquake hit. This was caused by the buffer effect of stocking components and spare parts. Except this buffer, the disruption in and recovery of the passenger vehicles sector by disaster are simulated fairly well in Fig. 5.

\section{Discussion}

We found that the disruption in the supply of semiconductor parts and rechargeable batteries in Kanto area primarily affected the restoration rate. These days, semiconductor parts perform major functions in cars such as in transmissions and brakes, engines, fuel distribution systems, in-care entertainment systems, etc. Semiconductors are important for fuel regulation systems, acting in the anti-pollution and fuel efficiency roles. Given the close relationships between the various industries, a total disruption in a low-tier industry can cause the same in a higher-tier one.

Immediately after the disaster, the power and communications blackout shut all business operations even if no actual damage was experienced. We can simulate this by setting the transaction values for the transport sector as zero. Further, though excess capacity in other regions ensured adequate replacements for some other items, transportation remained an issue.

The affected plants took six months to reach pre-disaster production levels, primarily because both restoring production in the semiconductor plants and ensuring replacements in these sectors turned out to be cumbersome affairs. 
While our simulation reproduced fairly well the disruption in and restoration of the automotive industry, our model did not consider real buffer effects thereby deeming the immediate post-disaster production values to be nil. We plan to investigate the buffer effects in the future and thus do not discuss these here.

\section{Conclusion}

From a review of the reports and news on the disruption and restoration process, we get that the restoration time of each supplier-sector in the supply chain varied with industrial characteristics. We also get that the limited/non-availability of sectors with long restoration time created ripples over the entire supply chain. We have simulated this ripple effect using the regional I-O table.

We also note that the intermediate table of the I-O table is a matrix showing the trade between demand-sectors and supply-sectors, and thus develop the supply-chain tier structure. Using the tier structure of the regional I-O table, we were able to specify the disaster attack point and vary the availability profile for each bottleneck sector. Using this 3-D matrix and the recurrence equation, we quantitatively estimate the indirect loss of production in the entire supply chain as a function of time.

Thus, this method can ascertain the indirect loss of production in a supply chain due to bottle-neck sectors. Our method can be utilized for priority decision making during/post disasters and for the economic evaluation of the effectiveness of preventive action for improving supply-chain resiliency.

The I-O table is often used in various business fields. We found that it can also be used to easily ascertain the industrial structure and the key processes from the view point of supply chain resiliency. Governments and industries can put this method to formulate a policy that prioritizes disaster management.

Acknowledgments. This work was supported in part by a Grant in Aid from the Global Center of Excellence Program for "Center for Education and Research of Symbiotic, Safe and Secure System Design" from the ministry of Education, Culture, Sport, and Technology in Japan.

\section{References}

1. Kouvelis, P., Chambers, C., Wang, H.: Supply Chain Management Research and Production and Operations Management: Review, Trends, and Opportunities. Production and Operations Management 15(3), 449-469 (2006)

2. Inoue, Y.: A Comprehensive Study on Research Approaches to Supply Chain Risk Identification. Shogaku Shushi 82(1), 45-60 (2012)

3. The Nikkei, The Asahi, The MID Japan Economist, and Nikkan Jidosha Newspaper

4. Asset securities reports of automotive manufacturers and reports by Japan Automobile Manufacturers Association

5. Trends in the automotive industry (manufacturer financial reports) 
6. Tokui, et al.: Effects of the Great East Japan Earthquake on Economics, RIETI Policy Discussion Paper Series 12-P-004 (March 2012)

7. Dick, et al.: Japan's 2011 Earthquake and Tsunami: Economic Effects and Implications for the United States, CSR Report for Congress (April 20, 2011)

8. Walid, K.: Modeling Approaches for the Design of Resilient Supply Networks under Disruption. International Journal Production Economics 135(2), 882-898 (2012)

9. 2005 Inter-Regional Input-Output Tables (sectors 12, 29, and 53), Ministry of Economy, Trade and Industry (March 26, 2010)

10. Stewart, R., Stone, J.B., Streitwieser, M.L.: U.S. Benchmark Input-Output Accounts, 2002. Survey of Current Business, 19-48 (2007)

11. Andrew, R., Glen, P.: A Multi-Region Input-Output Table Based on the Global Trade Analysis Project Database (GTAP-MRIO). Economic Systems Research 25(1), 99-121 (2013)

12. Hallegatte, S.: An Adaptive Regional Input-Output Model and its Application to the Assessment of the Economic Cost of Katrina. Risk Analysis: An International Journal 28(3), 779-799 (2008)

13. Yamano, N., Kajitani, Y., Shumuta, Y.: Modeling the Regional Economic Loss of Natural Disasters: The Search for Economic Hotspots. Economic Systems Research 19(2), 163 181 (2007)

14. Anderson, C.W., Santos, J.R., Haimes, Y.Y.: A Risk-based Input-Output Methodology for Measuring the Effects of the Northeast Blackout. Economic Systems Research 19(2), 183204 (2007) 